\title{
Grupos de doenças em trabalhadores do gênero masculino de uma universidade pública
}

\author{
Groups of diseases among public university male workers
}

\author{
Gabriela Berchiol Vieira ${ }^{1,2}$, Júlia Trevisan Martins ${ }^{1,3}$, Maria Cristina Cescatto Bobroff ${ }^{1,4} \bowtie$, Alexandrina Maciel Cardelli ${ }^{1,5}$, \\ Maria Lucia do Carmo Cruz Robazzi ${ }^{6}$, Gabriela Schmitt Trevisan ${ }^{7}$ \\ ${ }^{1}$ Universidade Estadual de Londrina (UEL), Londrina, PR \\ 2 Enfermeira, especialista em Saúde da Família, Mestre em Enfermagem pela UEL. \\ ${ }^{3}$ Enfermeira, Doutora em Enfermagem pela Universidade de São Paulo (USP) em Ribeirão Preto, Professora Adjunta da UEL. \\ ${ }^{4}$ Enfermeira, Doutora em Ciências da Saúde pela UEL, Professora Adjunta da UEL. \\ 5 Enfermeira, Doutora em Saúde Pública pela USP e London School of Hygiene And Tropical Medicine, Professora Associada da UEL. \\ ${ }^{6}$ Enfermeira, Doutora em Enfermagem pela USP, Professora Titular da Escola de Enfermagem da USP em Ribeirão Preto. Centro Colaborador \\ da Organização Mundial da Saúde para o Desenvolvimento da Pesquisa em Enfermagem, Ribeirão Preto, SP. \\ Estudante de Medicina da Universidade do Oeste Paulista, Presidente Prudente, SP.
}

\section{RESUMO}

Objetivos: Descrever os principais grupos de doenças em trabalhadores do gênero masculino atendidos na divisão ambulatorial de uma universidade pública.

Métodos: Estudo descritivo e retrospectivo com dados coletados de prontuários de consultas médicas realizadas no ano de 2009 , no ambulatório da Divisão de Assistência à Comunidade da Universidade Estadual de Londrina, em Londrina, estado do Paraná. Os diagnósticos médicos foram agrupados e categorizados conforme a Classificação Estatística Internacional de Doenças e Problemas Relacionados à Saúde (CID-10). A amostragem foi probabilística sistematizada, mantendo índice de confiabilidade de $95 \%$ e margem de erro de $5 \%$. Os resultados foram apresentados em números absolutos e frequências.

Resultados: Entre os 367 homens atendidos no período cujos prontuários foram selecionados para estudo, a faixa etária mais frequente foi acima dos 50 anos, havendo predomínio de auxiliares operacionais (67\%). O tempo de atuação na instituição superior a 11 anos foi o mais encontrado na amostra estudada. As doenças mais frequentemente descritas foram as do aparelho respiratório, com 66 casos (17,98\%), seguidas por doenças do aparelho circulatório com 58 casos (15,80\%), sistema osteomuscular e tecido conjuntivo com 51 casos (13,90\%), aparelho geniturinário com 45 casos $(12,26 \%)$ e transtornos mentais e comportamentais com 40 casos $(10,90 \%)$.

Conclusões: Constatou-se que muitas das doenças que acometeram os homens que trabalhavam na Universidade Estadual de Londrina são passíveis de prevenção. Os resultados deste estudo podem contribuir para o planejamento de ações que visem à promoção da saúde e à prevenção de agravos, especificamente voltadas para a população de trabalhadores do gênero masculino.

DESCRITORES: saúde do trabalhador; saúde do homem; assistência ambulatorial.

\section{ABSTRACT}

Aims: To describe the main groups of diseases in male workers treated at an outpatient clinic of a public university.

Methods: Descriptive and retrospective study with data collected from records of medical examinations carried out at the outpatient clinic of the Division of Community Healthcare of Universidade Estadual de Londrina, in the state of Paraná, Brazil, in 2009. The medical diagnoses were grouped and categorized according to the International Statistical Classification of Diseases and Related Health Problems (CID-10). A systematic sampling method, with a $95 \%$ confidence interval and a margin of error of $5 \%$, was used. The results were expressed as absolute numbers and frequencies.

Results: There was a predominance of men aged over 50 years, and those working as operations assistants accounted for $67 \%$ of the sample. Duration of employment was over 11 years in most cases. The most frequent groups of diseases were those which affected the respiratory tract (66 cases or $17.98 \%$ ), the circulatory system (58 cases or $15.80 \%)$, musculoskeletal and connective tissues (51 cases or $13.90 \%)$, the genitourinary tract (45 cases or 12.26\%), including also mental and behavioral disorders (40 cases or 10.90\%).

Conclusions: Most of the diseases that affected the men in this study are preventable. The results herein contribute towards the planning of health promotion and prevention actions, especially targeted at male workers.

KEY WORDS: occupational health; men's health; ambulatory care. 


\section{INTRODUÇÃO}

Os primeiros estudos sobre a saúde de homens surgiram em meados de 1970 nos Estados Unidos e estavam voltados para os déficits de saúde. A partir da década de 1990 a discussão deste tema passou a refletir uma perspectiva integral à saúde dos homens. No Brasil em 2005, este tema esteve em foco no âmbito da Saúde Coletiva, quando se elucidava o processo saúde-doença e a masculinidade, a fim de propor o enfrentamento do adoecimento e a promoção da saúde dessa população [1].

Porém, somente em 2008 o Ministério da Saúde brasileiro lançou a Política Nacional de Atenção Integral à Saúde do Homem tendo como principal objetivo entender a realidade masculina nos seus diversos contextos socioculturais e político-econômicos para, assim, promover ações de saúde que possibilitassem o aumento da expectativa de vida e a redução dos índices de morbimortalidade por causas preveníveis e evitáveis nessa população [2].

Desde então, o gênero masculino tem sido alvo de maior visibilidade da mesma maneira que outros grupos com focos mais antigos de atenção à saúde. Entretanto, um dos grandes obstáculos à promoção da saúde dos homens consiste na ideia de invulnerabilidade na construção masculina hegemônica. Na grande maioria das vezes, os homens procuram os serviços de saúde de forma episódica e por motivos específicos, quando têm dores que se tornam insuportáveis ou quando há impossibilidade de trabalhar, pois se sentem incomodados com a situação passiva e dependente do papel de doente, resultando muitas vezes na prática de automedicação $[3,4]$.

Pesquisadores brasileiros identificaram as causas da baixa procura dos homens por serviços de saúde, citando-se o modelo hegemônico de masculinidade imposto pela cultura, isto é, eles precisam ser viris e fortes, e buscar medidas preventivas seria uma forma de demostrar fraqueza; a falta de tempo; a dificuldade ou impossibilidade de deixar as atividades; o medo da revelação de uma doença grave; a vergonha de mostrar o corpo; as dificuldades de compatibilizar os horários de atendimento das Unidades Básicas de Saúde (UBS) com o trabalho [5].

Enfatiza-se que os estudos envolvendo os problemas de saúde dos homens têm aumentado ao longo do tempo, o que tem ajudado a dar visibilidade sobre o adoecimento e agravos dos mesmos. Contudo, pesquisas sobre as doenças que atingem o trabalhador masculino ainda são incipientes [6].

Considera-se relevante uma investigação que aborde o adoecimento dos trabalhadores do gênero masculino, uma vez que os resultados poderão contribuir para a programação de ações de promoção à saúde, prevenção de doenças e diminuição dos agravos à saúde, visando a uma melhor qualidade de vida para os homens. Assim, este estudo teve como objetivo descrever os principais grupos de doenças dos trabalhadores do gênero masculino atendidos na divisão ambulatorial de uma universidade pública.

\section{MÉTODOS}

Este estudo compreende uma pesquisa descritiva e retrospectiva, realizada com dados de prontuários de atendimento ambulatorial da Divisão de Assistência à Comunidade (DASC), da Universidade Estadual de Londrina (UEL), estado do Paraná.

$\mathrm{Na}$ DASC presta-se assistência ambulatorial de atenção primária por meio de atendimentos eletivos ou de pronto atendimento aos trabalhadores e estudantes da UEL. Essa divisão tem como finalidade o desenvolvimento de ações de promoção, proteção e tratamento à saúde por meio de atendimentos médicos nas áreas de clinica médica (cardiologia, urologia, oftalmologia, psiquiatria e ginecologia), clínica odontológica e enfermagem. Compõem a equipe multiprofissional da divisão 44 profissionais administrativos, de enfermagem (enfermeiros, técnicos e auxiliares), odontologia e medicina. Os atendimentos são realizados de segunda a sexta-feira ente $07 \mathrm{e}$ 22 horas, tanto por agendamento como em pronto atendimento.

Considerando o número de prontuários de atendimentos/consultas médicas realizadas no ano de 2009, estabeleceu-se a amostra adotando-se os seguintes procedimentos: a) identificação do total geral de atendimentos $(n=13.689)$; b) identificação do total de atendimentos de trabalhadores $(\mathrm{n}=3.418)$; c) identificação do total de atendimentos de trabalhadores do gênero masculino ( $\mathrm{n}=1.570)$; d) definição da amostra probabilística, de forma sistematizada, mantendo o índice de confiabilidade de $95 \%$ e margem de erro de 5\% ( $n=367)$. Assim, a amostra constou de 367 prontuários relativos aos atendimentos/consultas médicas de trabalhadores do gênero masculino nas especialidades de clínica médica, cardiologia e urologia durante o ano de 2009. Essas três especialidades foram as que prestaram atendimentos para pessoas do gênero masculino em 2009, ano em que não houve atendimentos nas áreas de psiquiatria e oftalmologia por falta de recursos humanos.

A amostra foi estabelecida por meio da seleção consecutiva de um a cada quatro prontuários. Como 
critérios de inclusão dos prontuários estabeleceu-se que o paciente deveria ser do gênero masculino, pertencer ao quadro de funcionários da UEL e as anotações referentes a cada atendimento deveriam descrever o diagnóstico médico e apresentar letra legível. Dessa forma, os prontuários de estudantes, tanto de graduação quanto de pós-graduação, não foram incluídos.

As variáveis coletadas foram dados sociodemográficos (idade, categoria profissional, função desempenhada e tempo de serviço) e diagnóstico médico. A função desempenhada foi agrupada de acordo com os termos utilizados pela Pró-Reitoria de Recursos Humanos da UEL (Quadro 1). O diagnóstico médico foi agrupado e categorizado conforme a Classificação Estatística Internacional de Doenças e Problemas Relacionados à Saúde (CID-10) [7].

Quadro 1. Ocupações e funções, conforme a classificação utilizada pela Universidade Estadual de Londrina, que apareceram na amostra de 367 prontuários de trabalhadores do gênero masculino selecionados para estudo. Londrina, PR, Brasil, 2009.

\begin{tabular}{|c|c|}
\hline Categorias & Funções \\
\hline $\begin{array}{l}\text { Auxiliar } \\
\text { operacional }\end{array}$ & $\begin{array}{l}\text { Motorista, vigia, servente, zelador, auxiliar } \\
\text { de serviços gerais, técnico em manutenção, } \\
\text { auxiliar de produção agropecuária, operador de } \\
\text { equipamentos, auxiliar de biblioteca, auxiliar } \\
\text { administrativo, carpinteiro, segurança, pedreiro, } \\
\text { serralheiro, assistente de agronomia, porteiro, } \\
\text { operador de rádio, encanador, jardineiro, auxiliar } \\
\text { de reprografia, almoxarife, técnico de assuntos } \\
\text { universitários, auxiliar em eletrônica, operador } \\
\text { gráfico, contínuo. }\end{array}$ \\
\hline $\begin{array}{l}\text { Técnico } \\
\text { Administrativo }\end{array}$ & $\begin{array}{l}\text { Técnico de informática, técnico de radiologia, } \\
\text { técnico de laboratório, técnico agrícola, técnico } \\
\text { de produção industrial, escriturário, técnico em } \\
\text { odontologia, técnico de agronomia, técnico em } \\
\text { projetos visuais, técnico de enfermagem, auxiliar } \\
\text { administrativo, desenhista projetista, analista de } \\
\text { informática, atendente de veterinária, locutor, } \\
\text { auxiliar de nutrição, auxiliar de enfermagem, } \\
\text { topógrafo, auxiliar de laboratório, auxiliar de } \\
\text { agropecuária. }\end{array}$ \\
\hline Docente & Biólogo, odontólogo, físico, bioquímico \\
\hline
\end{tabular}

O banco de dados foi construído no programa Microsoft Excel 2010 e analisado no programa IBM-SPSS versão 19.0. A análise consistiu no cálculo das frequências em números absolutos e relativos.

Os aspectos éticos de pesquisa em seres humanos foram respeitados conforme o Conselho Nacional de Saúde do Brasil, e o projeto de pesquisa foi aprovado pelo Comitê de Ética em Pesquisa Envolvendo Seres Humanos da UEL, sob o parecer consubstanciado de número 295/10.

\section{RESULTADOS}

No ano de 2009 constavam no quadro funcional da UEL 5.547 servidores. Entre estes, eram 2.976 mulheres e 2.571 homens. No mesmo ano, foram realizados 13.689 atendimentos pela DASC, sendo 10.271 para alunos e 3.418 para servidores. Entre estes últimos, foram atendidas 1.848 (54\%) mulheres e 1.570 (46\%) homens (população deste estudo).

Entre os 367 prontuários de atendimentos/consultas médicas da amostra analisada, a idade média dos homens foi de $50 \pm 10$ anos. A faixa etária mais numerosa foi entre 51 e 60 anos ( $n=140 ; 38,2 \%)$, seguida das faixas etárias 41 a 50 anos $(\mathrm{n}=123 ; 33,5 \%), 31$ a 40 anos $(n=50 ; 13,6 \%), 61$ a 70 anos $(n=44 ; 12,0 \%)$ e com até 30 anos de idade $(n=10 ; 2,7 \%)$. O tempo médio de trabalho na universidade foi de $21 \pm 7$ anos, sendo que $50 \%$ dos trabalhadores tinham tempo de trabalho de 20 anos ou mais e $50 \%$ abaixo de 20 anos.

A categoria profissional denominada auxiliar operacional foi a mais frequente, com 246 atendimentos $(67,03 \%)$, seguida pela categoria técnico administrativo, com 71 atendimentos $(19,35 \%)$ e, por último, pela categoria docente, que incluiu biólogos, odontólogos, físicos e bioquímicos, com 23 atendimentos (6,27\%). Houve ainda alguns prontuários nos quais não constava a categoria do trabalhador $(\mathrm{n}=27 ; 7,35 \%)$ e que foram considerados como ignorados.

Entre as doenças classificadas de acordo com a CID10 , houve predomínio das doenças do aparelho respiratório e circulatório na amostra estudada (Tabela 1).

Tabela 1. Distribuição dos atendimentos/consulta médica no ambulatório da Divisão de Assistência à Comunidade da Universidade Estadual de Londrina, segundo as Famílias de Doenças da CID-10. Londrina, PR, Brasil, 2009.

\begin{tabular}{|c|c|c|}
\hline Famílias de Doenças & $\mathrm{n}$ & $(\%)$ \\
\hline Doenças do aparelho respiratório & 66 & $(17,98)$ \\
\hline Doenças do aparelho circulatório & 58 & $(15,80)$ \\
\hline Doenças do sistema osteomuscular e do tecido conjuntivo & 51 & $(13,90)$ \\
\hline Doenças do aparelho geniturinário & 45 & $(12,26)$ \\
\hline Transtornos mentais e comportamentais & 40 & $(10,90)$ \\
\hline Doenças do aparelho digestivo & 31 & $(8,45)$ \\
\hline Doenças endócrinas, nutricionais e metabólicas & 24 & $(6,54)$ \\
\hline Doenças do olho e anexos & 14 & $(3,81)$ \\
\hline Doenças da pele e do tecido subcutâneo & 12 & $(3,27)$ \\
\hline Doenças do sistema nervoso & 10 & $(2,72)$ \\
\hline Doenças do ouvido e da apófise mastoide & 7 & $(1,91)$ \\
\hline Lesões, envenenamento e consequências de causas externas & 6 & $(1,63)$ \\
\hline Algumas doenças infecciosas e parasitárias & 2 & $(0,54)$ \\
\hline Neoplasias & 1 & $(0,27)$ \\
\hline Total da amostra de prontuários estudados & 367 & $(100)$ \\
\hline
\end{tabular}


Tabela 2. Distribuição das comorbidades em uma amostra de 367 atendimentos/consulta médica de trabalhadores do gênero masculino no ambulatório da Divisão de Assistência à Comunidade da Universidade Estadual de Londrina, de acordo com a ocupação. Londrina, PR, Brasil, 2009.

\begin{tabular}{|c|c|c|c|c|c|}
\hline \multirow[t]{2}{*}{ Comorbidades } & $\begin{array}{c}\text { Auxiliar } \\
\text { Operacional }\end{array}$ & Docente & Ignorado & $\begin{array}{c}\text { Técnico } \\
\text { Administrativo }\end{array}$ & Total \\
\hline & n (\%) & n (\%) & n (\%) & n (\%) & n (\%) \\
\hline HAS & $59(24,00)$ & $06(26,10)$ & $11(40,74)$ & $22(30,99)$ & $98(26,70)$ \\
\hline Transtorno psiquiátrico & $26(10,56)$ & $02(8,69)$ & - & $09(12,68)$ & $37(10,08)$ \\
\hline HAS/Transtorno psiquiátrico & $28(11,38)$ & - & - & - & $28(7,63)$ \\
\hline $\mathrm{HAS} / \mathrm{DM}$ & $21(8,53)$ & $02(8,69)$ & - & - & $23(6,26)$ \\
\hline HAS/Dislipidemia & $17(6,91)$ & $02(8,69)$ & - & - & $19(5,18)$ \\
\hline HAS/DM/Outros agravos & $11(4,48)$ & $02(8,69)$ & $02(7,41)$ & $02(2,81)$ & $17(4,63)$ \\
\hline HAS/Obesidade & $09(3,66)$ & - & - & $06(8,45)$ & $15(4,09)$ \\
\hline Dislipidemia & - & $09(39,14)$ & $04(14,81)$ & - & $13(3,54)$ \\
\hline DM & - & - & $04(14,81)$ & - & $04(1,09)$ \\
\hline Outras* & $75(30,48)$ & - & $06(22,23)$ & $32(45,07)$ & $113(30,80)$ \\
\hline TOTAL & $246(100)$ & $23(100)$ & $27(100)$ & $71(100)$ & $367(100)$ \\
\hline
\end{tabular}

HAS: hipertensão arterial sistêmica; DM: diabetes mellitus.

*Outras: arritmia cardíaca, bronquite, doença de Chagas, convulsão, esteatose hepática, etilismo, hepatopatia crônica, ascite, gastrite, nefrolitíase, pancreatite, hipertrofia prostática benigna, labirintite.

Excluindo-se a categoria "outras", a comorbidade mais frequentemente identificada entre os trabalhadores atendidos na DASC foi a hipertensão arterial sistêmica (HAS), associada ou não a outras comorbidades. Essa comorbidade foi também a mais frequente entre os auxiliares operacionais e técnicos administrativos, enquanto entre os docentes, a comorbidade mais detectada foi a dislipidemia (Tabela 2).

\section{DISCUSSÃO}

Apesar de que o foco deste estudo foi descrever os principais grupos de doenças dos trabalhadores do gênero masculino e não a profissão, observou-se que a maioria dos trabalhadores atendidos na DASC pertencia ao quadro de servidores denominados como auxiliar operacional. Considera-se que a escolha do método de pesquisa retrospectiva e a utilização de dados registrados nos prontuários podem ter limitado alguns resultados e inviabilizado algumas comparações. Um estudo da mesma natureza em caráter prospectivo poderia contribuir com dados que pudessem ser correlacionados aos resultados da presente pesquisa.

A categorização dos trabalhadores em razão de seus cargos é importante visto que as distintas condições de jornadas de trabalho, a função exercida e a remuneração estão diretamente relacionadas à manutenção de alimentação adequada, exercícios físicos contínuos, boas condições de moradia e acesso aos serviços de saúde, determinantes que podem causar impactos decisivos na saúde [8-9].
No presente estudo verificou-se um número expressivo de pacientes com HAS como comorbidade, assim como de outras comorbidades associadas à HAS. Resultados similares foram encontrados em estudo que identificou a HAS como a doença com maior prevalência nos trabalhadores de um Centro de Saúde Escola, associada principalmente à obesidade, sobretudo no gênero masculino [10], assim como em outra investigação que demonstrou a prevalência de HAS em 45,9\% dos servidores de gênero masculino em uma universidade pública do Distrito Federal [11].

No Brasil, assim como em outros países da América Latina, observou-se, nas últimas décadas, uma importante mudança no perfil da morbidade da população, no qual as doenças cardiovasculares e a HAS destacaram-se com uma prevalência estimada de $35 \%$ na população acima de 40 anos [12]. Embora a constatação da relação entre a ocorrência de doenças crônico-degenerativas e o trabalho não ser fácil de comprovar, o aumento da prevalência dessas doenças e seu impacto na saúde dos trabalhadores é evidente nos dias atuais $[8,13]$.

Entre as famílias de doenças identificadas na presente pesquisa, as doenças do aparelho respiratório foram as causas de atendimentos/consultas médicas mais frequentes na DASC em 2009. Os problemas respiratórios são geralmente responsáveis por grande procura de consultas médicas em âmbito ambulatorial, bem como por serviços de emergência e hospitalização [14]. No que tange aos resultados deste estudo, infere-se que o grande número de atendimentos/ consultas médicas possa ser justificado pela pandemia 
de Influenza A H1N1 ocorrida no ano de 2009. O novo vírus influenza $\mathrm{A}$ que ocasionou casos de síndrome respiratória aguda foi identificado primeiramente no México e nos Estados Unidos da América e rapidamente se disseminou pelo mundo, atingindo também a população brasileira e chegando ao estado do Paraná em junho de 2009 [15].

Ademais, verificou-se elevado número de atendimentos por doenças do aparelho circulatório. Sabese que essas doenças têm prevalecido no perfil de morbimortalidade do gênero masculino. Esse novo perfil pode ser resultante das mudanças ocorridas na organização laboral, tais como o surgimento da informática, automação, restrição hierárquica, diminuição do efetivo, desemprego e sofrimento [8], além de condições de vida sedentária e dificuldades de alimentação adequada (valor nutricional, quantidade e qualidade dos alimentos, horários, hidratação, preços dos produtos, entre outros).

Ocorreu uma grande procura pelo atendimento/ consulta médica à DASC devido a problemas relacionados ao sistema osteomuscular e ao tecido conjuntivo. Dados da literatura mostram que a partir do ano 2000 tais doenças passaram a representar o principal grupo de agravos à saúde, entre as doenças ocupacionais brasileiras; afetam diferentes categorias profissionais e são conhecidas por diversas denominações, sendo as mais comuns lesões por esforços repetitivos e distúrbios osteomusculares relacionados ao traba1ho. Essas doenças provocam danos ao sistema osteomuscular principalmente oriundos da utilização excessiva de determinados grupos musculares, normalmente relacionada à atividade ocupacional, e à falta de tempo para recuperação do corpo. Os principais sintomas refereridos pelas pessoas acometidas por esses problemas são dor, parestesia, sensação de peso e fadiga principalmente nos membros superiores [16].

Em pesquisa realizada com trabalhadores de indústria metalúrgica do município de Canoas, RS, a associação do perfil sociodemográfico aos sintomas osteomusculares e ocupacionais demonstrou que $73,2 \%$ dos trabalhadores apresentaram algum tipo de sintoma osteomuscular nos 12 meses antecedentes ao estudo, sendo 21,35\% desses com afastamento do trabalho [17]. Em outra investigação, realizada com trabalhadores de uma industria da região sul do Brasil, houve predominio do gênero masculino e alta prevalência $(75,2 \%)$, nesse grupo, de algum tipo de sintoma osteomuscular como dor, desconforto ou dormência [16]. Pesquisa com professores universitários da cidade de Belo Horizonte, MG, revelou que as doenças do sistema osteomuscular ocuparam o terceiro lugar em dias de afastamento do trabalho, correspondendo a $11,5 \%$ [18].

Outras pesquisas têm demonstrado limitações físicas e psicossociais em decorrência de doenças relacionadas diretamente ao sistema músculoesquelético. Como exemplos citam-se o afastamento do trabalho com consequente frustração e depressão por não se conseguir executar as atividades rotineiras. Além disso, há o preconceito e o constrangimento do profissional em ter que provar aos familiares, ao empregador e aos órgãos públicos a existência da doença $[19,20]$.

Com relação às doenças do aparelho geniturinário também houve uma procura considerável. Os homens buscam atendimento do urologista principalmente devido à hipertrofia prostática benigna, que atinge a maioria da população do gênero masculino após os 50 anos de idade e acarreta diversos sintomas urinários com impacto muito negativo na qualidade de vida dos mesmos [2]. Entretanto, embora os homens venham buscando cada vez mais atendimentos pela urologia, é fato, ainda, que enquanto 16,7 milhões de mulheres procuram assistência de um ginecologista, apenas 2,7 milhões daqueles consultam um urologista [3]. Uma hipótese para este fato é que os serviços de saúde têm uma lógica de atendimento voltada prioritariamente à saúde sexual e reprodutiva das mulheres, à gravidez e à contracepção, dispondo inclusive de profissionais especializados em ginecologia e obstetrícia para atendimento dessa demanda. Em contrapartida, notase que nos mesmos serviços de atenção primária são escassos os profissionais que cuidam especificamente de problemas sexuais e reprodutivos do gênero masculino [1].

A atenção à saúde do homem, particularmente em relação aos portadores de doenças sexualmente transmissíveis, foi abordada em estudo realizado em Fortaleza, CE. Os autores destacam a importância de políticas públicas voltadas para esse público e afirmam que "a inclusão do homem como uma das prioridades de atenção à saúde é urgente" [21].

Os transtornos mentais e comportamentais se configuraram como a quinta maior causa de atendimentos/consultas médicas na DASC. Dados de levantamento realizado pelo Ministério da Saúde, comparando os anos de 1995 e de 2005, demonstraram um aumento das internações hospitalares por transtornos mentais e comportamentais no Sistema Único de Saúde [22]. Além disso, outros pesquisadores salientam que, no ano 2020, as doenças mentais e comportamentais, principalmente a depressão, serão predominantes entre as principais causas de 
incapacidade no mundo, apenas perdendo para as doenças cardíacas isquêmicas, e advertem que $81,6 \%$ dos transtornos mentais identificados em profissionais correspondem a problemas depressivos/ansiosos frequentemente relacionados ao trabalho [13].

Alterações na saúde física e mental dos trabalhadores podem ser identificadas em decorrência do estresse desencadeado no ambiente laboral, ocasionando implicações no processo de trabalho tais como problemas de relacionamento interpessoal, absenteísmo, acidentes, insatisfação com o trabalho e morte [23]. A organização do labor tem atuado na gênese da fadiga física e do sofrimento mental. Jornadas de trabalho prolongadas, trabalhos em turno, especialmente noturno, ritmos acelerados de produção, divisão e padronização de tarefas, com subutilização da competência técnica e da criatividade, rigidez hierárquica e não reconhecimento profissional e pessoal são situações que desencadeiam o desgaste do trabalhador [24].

As doenças do aparelho digestivo também tiveram expressiva procura de atendimento na DASC, constituindo-se na sexta causa. Estudo brasileiro sobre internações hospitalares no setor público identificou as doenças do aparelho digestivo como a terceira causa das hospitalizações, advertindo que há necessidade de os gestores públicos criarem estratégias que visem à prevenção dessas doenças [25].

Denota-se a relevância dos resultados deste estudo, porém devem ser consideradas algumas limitações devido às restrições da abordagem metodológica empregada. O estudo retrospectivo realizado em uma única instituição, durante um período limitado de tempo, bem como a falta de alguns dados nos prontuários, restringe análises comparativas com outras investigações, além da escassez de pesquisa nacional e internacional com a temática saúde e doença no gênero masculino.

Apesar das limitações, os resultados deste estudo revelam uma visão abrangente sobre as doenças que acometeram trabalhadores do gênero masculino. Verificou-se que muitas das doenças encontradas nos homens são passíveis de prevenção. Desta forma, este estudo propicia subsídios aos gestores no planejamento da atenção específica à população de trabalhadores do gênero masculino.

\section{REFERÊNCIAS}

1. Gomes R, Moreira MCN, Nascimento EF, Sousa Rebello LEFS, Couto MT, Schraiber LB. Os homens não vêm! Ausência e/ou invisibilidade masculina na atenção primária. Ciênc Saúde Coletiva. 2011;16(suppl.1):983-92. http://dx.doi.org/10.1590/S1413-81232011000700030

2. Brasil. Ministério da Saúde. Política nacional de atenção integral à saúde do homem: princípios e diretrizes. Brasília, DF: OPAS; 2009. [Cited 2015 Jan] Available from: http://portalsaude.saude.gov.br/images/pdf/2014/maio/21/CNSH-DOC-PNAISH---Principios-e-Diretrizes. pdf

3. Carrara S, Russo JA, Faro L. A política de atenção à saúde do homem no Brasil: os paradoxos da medicalização do corpo masculino. Physis. 2009;19(3):659-78. http://dx.doi.org/10.1590/S0103-73312009000300006

4. Gomes R, Nascimento EF, Araújo FC. Por que os homens buscam menos os serviços de saúde do que as mulheres? As explicações de homens com baixa escolaridade e homens com ensino superior. Cad Saúde Pública. 2007;23(3):565-74. http://dx.doi.org/10.1590/S0102$311 X 2007000300015$

5. Gomes R, Schraiber LB, Couto MT. Homens e saúde na pauta da Saúde Coletiva. Ciênc Saúde Coletiva. 2005;10(1):7-17. http://dx.doi. org/10.1590/S1413-81232005000100002

6. Courtenay WH. Constructions of masculinity and their influence on men's well-being: a theory of gender and health. Soc Sci Med. 2000; 50 (10):1385-401. http://dx.doi.org/10.1016/S0277-9536(99)00390-1

7. Organização Mundial da Saúde. Classificação estatística internacional de doenças e problemas relacionados à saúde - CID-10. $8^{\mathrm{a}}$ ed. São Paulo, SP: EDUSP; 2000.

8. Cunha JB, Blank VLG, Boing AF. Tendência temporal de afastamento do trabalho em servidores públicos (1995-2005). Rev Bras Epidemiol. 2009;12(2):226-36. http://dx.doi.org/10.1590/S1415-790X2009000200012

9. Silveira SG, Grisotti M. Trabalho e saúde: um estudo sobre o processo saúde-doença dos servidores de um hospital universitário. Sau \& Transf Soc. 2011;2(1):18-27.

10. Nascimento LC, Mendes IJM. Perfil de saúde dos trabalhadores de um Centro de Saúde-Escola. Rev Latinoam Enferm. 2002;10 (4):502-8. http://dx.doi.org/10.1590/S0104-11692002000400006

11. Conceição TV, Gomes FA, Tauil PL, Rosa TT. Valores de pressão arterial e suas associações com fatores de risco cardiovasculares em servidores da Universidade de Brasília. Arq Bras Cardiol. 2006;86(1):26-31. http://dx.doi.org/10.1590/S0066-782X2006000100005

12. Ribeiro AG, Cotta RM, Ribeiro SMR. A Promoção da saúde e a prevenção integrada dos fatores de risco para doenças cardiovasculares. Ciênc Saúde Coletiva. 2012;17(1):7-17. http://dx.doi.org/10.1590/S1413-81232012000100002

13. Andrade TB, Souza MGC, Simões MPC, Andrade FB. Prevalência de absenteísmo entre trabalhadores do serviço público. Sci Med. 2008;18(4):166-71. 
14. Greenberg SB. Respiratory viral infections in adults. Curr Opin Pulm Med. 2002;8(3):201-8. http://dx.doi.org/10.1097/00063198200205000-00009

15. Duarte PAD, Venazzi A, Youssef NCM, Oliveira MC, Tannous LA, Duarte CB, Grion CMC, Germano A, Schiavetto PM, Lins ALGP, Campos MMF, Miúra CK, Bredt CSO, Toso LC, Réa-Neto A. Outcome of influenza A (H1N1) patients admitted to intensive care units in the Paraná state, Brazil. Rev Bras Ter Intensiva. 2009;21(3):231-6. http://dx.doi.org/10.1590/S0103-507X2009000300001

16. Trelha CS, Cunha, ACV, Silva DW, Lopes AR, Parra KC, Citadini JM, Gallo DLL, Nakano MM, Castro RFD, Carregaro RL. LER/DORT em operadores de checkout: um estudo de prevalência. Salusvita. 2002; 21(3):87-95.

17. Picoloto D; Silveira E. Prevalência de sintomas osteomusculares e fatores associados em trabalhadores de uma indústria metalúrgica de Canoas, RS. Ciênc Saúde Coletiva. 2008;13(2):507-16. http://dx.doi.org/10.1590/S1413-81232008000200026

18. Gasparini SM, Barreto MS, Assunção AA. O professor, as condições de trabalho e os efeitos sobre sua saúde. Educação e Pesquisa. 2005;31(2):189-99. http://dx.doi.org/10.1590/S1517-97022005000200003

19. Lima MFEM, Lima-Filho DO. Condições de trabalho e saúde do/a professor/a universitário/a. Cien Cogn. 2009;14(3):62-82.

20. Rodrigues BC, Moreira CCC, Triana TA, Rabelo JF, Higarashi IH. Limitações e consequências na vida do trabalhador ocasionadas por doenças relacionadas ao trabalho. Rev Rene. 2013;14(2):448-57.

21. Souza AR, Freitas APC, Rovere GP, Moura ADA, Feitoza AR. Perfil de usuários masculinos atendidos em um serviço de referência para doenças sexualmente transmissíveis. Rev Rene. 2012;13(4):734-43.

22. Rede Interagencial de Informação para a Saúde (RIPSA). Indicadores básicos para a saúde no Brasil: conceitos e aplicações/RIPSA. 2ª ed Brasília: Organização Pan-Americana da Saúde, 2008. [Cited 2015 Feb] Available from: http://tabnet.datasus.gov.br/tabdata/livroidb/2ed/ indicadores.pdf

23. Robazzi MLCC, Mauro MYC, Dalri RCMB, Silva LA, Secco IAO, Pedrão LJ. Exceso de trabajo y agravios mentales a los trabajadores de la salud. Rev Cuba Enferm. 2010;26(1):52-64.

24. Martins JT, Robazzi MLCC, Bobroff MCC. Prazer e sofrimento no trabalho da equipe de enfermagem: reflexão à luz da psicodinâmica Dejouriana. Rev Esc Enferm USP. 2010;44(4):1107-11. http://dx.doi.org/10.1590/S0080-62342010000400036

25. Lima e Costa MFF, Guerra HL, Barreto SM, Guimarães RM. Diagnóstico da situação da população idosa brasileira: um estudo da mortalidade e das internações hospitalares públicas. Inf Epidemiol SUS. 2000; 9(1):43-50. http://dx.doi.org/10.5123/S0104-16732000000100003 